\title{
Automated Axon Tracking of 3D Confocal Laser Scanning Microscopy Images Using Guided Probabilistic Region Merging
}

\author{
Ranga Srinivasan • Xiaobo Zhou • Eric Miller • Ju Lu • \\ Jeff W. Lichtman • Stephen T. C. Wong
}

Published online: 8 January 2009

(C) Humana Press Inc. 2009

\section{Erratum to: Neuroinform (2007) 5:189-203}

DOI 10.1007/s12021-007-0013-4

Due to a production error an author's name was misspelled. The correct spelling is Lichtman. We apologize for the error.

The online version of the original article can be found at: http://dx.doi. org/10.1007/s12021-007-0013-4.

R. Srinivasan $\cdot$ X. Zhou $\cdot$ S. T. C. Wong Harvard Center for Neurodegeneration and Repair-Center for Bioinformatics, Harvard Medical School,

Boston, MA, USA

R. Srinivasan

Department of Electrical and Computer Engineering,

Northeastern University,

Boston, MA, USA

E. Miller

Department of Electrical and Computer Engineering,

Tufts University,

Medford, MA, USA

\section{Zhou $\cdot$ S. T. C. Wong $(\bowtie)$}

Functional and Molecular Imaging Center,

Department of Radiology, Brigham and Women's Hospital and Harvard Medical School,

Boston, MA, USA

e-mail: wong@crystal.harvard.edu

J. Lu $\cdot$ J. W. Lichtman

Department of Molecular and Cellular Biology,

Harvard University,

Cambridge, MA, USA 